\title{
Randomized, crossover questionnaire survey of acceptabilities of controlled-release mesalazine tablets and granules in ulcerative colitis patients
}

\author{
Keiji Yagisawa ${ }^{1}$, Taku Kobayashi², Ryo Ozaki², Shinji Okabayashi², Takahiko Toyonaga ${ }^{2}$, Miki Miura ${ }^{3}$, Mari Hayashida ${ }^{3}$, \\ Eiko Saito ${ }^{2}$, Masaru Nakano ${ }^{2}$, Hajime Matsubara ${ }^{1}$, Tadakazu Hisamatsu' ${ }^{3}$, Toshifumi Hibi ${ }^{2}$ \\ ${ }^{I}$ Department of Pharmacy and ${ }^{2}$ Center for Advanced IBD Research and Treatment, Kitasato University Kitasato Institute Hospital, Tokyo; \\ ${ }^{3}$ The Third Department of Internal Medicine, Kyorin University School of Medicine, Tokyo, Japan
}

Background/Aims: Oral mesalazine is an important treatment for ulcerative colitis (UC), and non-adherence to mesalazine increases the risk of relapse. Controlled-release (CR) mesalazine has 2 formulations: tablets and granules. The relative acceptabilities of these formulations may influence patient adherence; however, they have not been compared to date. This study aimed to evaluate the acceptabilities of the 2 formulations of CR mesalazine in relation to patient adherence using a crossover questionnaire survey. Methods: UC patients were randomly assigned to 2 groups in a 1:1 ratio. Patients in each group took either $4 \mathrm{~g}$ of CR mesalazine tablets or granules for 6 to 9 weeks, and then switched to $4 \mathrm{~g}$ of the other formulation for a further 6 to 9 weeks. The acceptability and efficacy were evaluated by questionnaires, and adherence was assessed using a visual analog scale. The difference in acceptabilities between the 2 formulations and its impact on adherence were assessed. Results: A total of 49 patients were prospectively enrolled and 33 patients were included in the analysis. Significantly more patients found the tablets to be less acceptable than the granules ( $76 \%$ vs. $33 \%, P=0.0005)$. The granules were preferable to the tablets when the 2 formulations were compared directly $(73 \%$ vs. $21 \%, P=0.004)$, for their portability, size, and numbers of pills. The adherence rate was slightly better among patients taking the granules (94\% vs. 91\%) during the observation period, but the difference was not significant $(P=0.139)$. Conclusion: $C R$ mesalazine granules are more acceptable than tablets, and may therefore be a better option for long-term medication. (Intest Res 2019;17:87-93)

Key Words: Colitis, ulcerative; Mesalamine; Medication adherence; Patient acceptance of health care; Drug compounding

\section{INTRODUCTION}

Ulcerative colitis (UC) is a life-long disorder of the colon, characterized by a relapsing-remitting course. ${ }^{1}$ The optimal goal of medical treatment in UC patients is to induce and maintain long-term remission. Oral 5-aminosalicylate (5-ASA) plays an

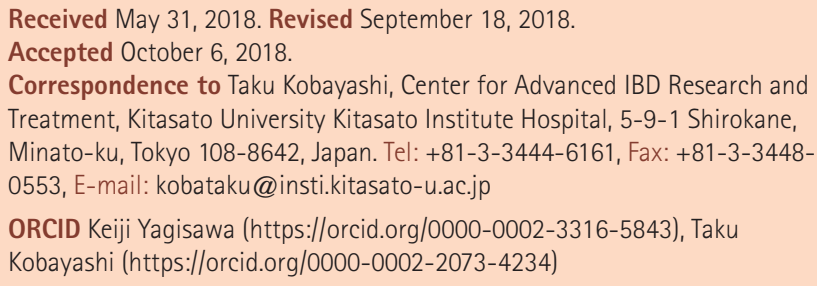

important role in both induction and maintenance therapies, and numerous studies have revealed that non-adherence to mesalazine is associated with an increased risk of clinical relapse. ${ }^{2,3}$ Improving adherence to mesalazine is therefore an important goal in daily clinical practice.

Ethylcellulose-coated controlled-release (CR) mesalazine $\left(\mathrm{PENTASA}^{\circledR}\right)$ is one of the major forms of oral 5-ASA. ${ }^{4,5} \mathrm{CR}$ mesalazine is available as 2 different formulations, tablets and granules, with no apparent difference in efficacy between them for UC, because both have the same mechanism of mesalazine release. ${ }^{6}$ However, the acceptabilities of the formulations may differ. In addition to the general differences between tablets and granules, CR mesalazine granules include a signifi- 
cantly lower percentage of additives compared with the tablets, while the numbers of tablets or sachets that need to be taken differ. It is therefore possible that these differences may influence patient adherence, ${ }^{7,8}$ however, no study has yet compared these 2 formulations in terms of acceptability and adherence.

This study aimed to evaluate the acceptabilities of the 2 formulations of CR mesalazine in relation to adherence among UC patients, using a crossover questionnaire survey.

\section{METHODS}

\section{Patients}

Outpatients diagnosed with UC at Kitasato University Kitasato Institute Hospital or Kyorin University Hospital and who were eligible for CR mesalazine were recruited from January to December 2016 in Kitasato University Kitasato Institute Hospital, and from April to August 2017 in Kyorin University Hospital. There was no age limit as long as the patients could assess the acceptability of the medications and answer the questionnaires unaided.

\section{Formulations of CR Mesalazine}

This crossover study compared PENTASA ${ }^{\circledR}$ tablets and granules. PENTASA ${ }^{\circledR}$ tablets (250 mg, $500 \mathrm{mg}$, and $1 \mathrm{~g}$ ) are approved in over 100 countries, and PENTASA ${ }^{\circledR}$ granules $(250 \mathrm{mg}, 500$ $\mathrm{mg}, 1 \mathrm{~g}, 2 \mathrm{~g}$, and $4 \mathrm{~g}$ ) are approved in over 80 countries worldwide. A single tablet contains about $33 \%$ additives, and a total weight of $6 \mathrm{~g}$ is therefore needed to deliver $4 \mathrm{~g}$ mesalazine, compared with only $4.24 \mathrm{~g}$ of the granules. PENTASA ${ }^{\circledR}$ tablets (500 mg/tablet) and granules ( $2 \mathrm{~g} / \mathrm{sachet})$ were used in this study.

\section{Study Design}

The outline of the study is shown in Fig. 1. Enrolled patients were randomly assigned to group 1 or group 2 in a 1:1 ratio. Patients in group 1 took CR mesalazine tablets and patients in group 2 took the granules for 6-9 $( \pm 3)$ weeks, and each group then switched to the other formulation for a further 6-9 $( \pm 3)$ weeks. Patients who were administered $2 \mathrm{~g}$ twice daily were further evaluated to compare the acceptability, adherence, and efficacy of the formulations. The endpoints of the study were the acceptability, preference, adherence, efficacy, and safety of the 2 formulations. Acceptability and preference were assessed based on the answers to the questionnaires, efficacy was assessed based on the changes of the partial Mayo score

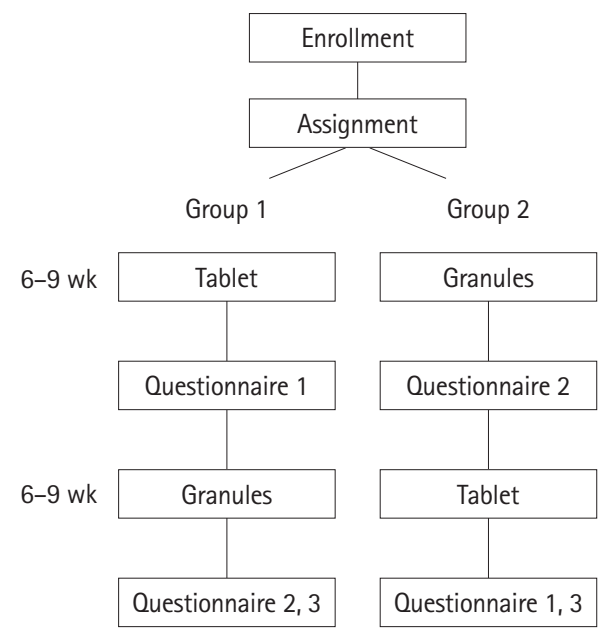

Fig. 1. Study design. Questionnaire 1, acceptability of tablets; questionnaire 2, acceptability of granules; questionnaire 3 , comparison of tablets and granules.

before and after taking each formulation and based on the answers to the questionnaires. The differences between the 2 formulations were evaluated. Adherence rate (\%) was assessed by a visual analog scale, ${ }^{9}$ with an adherence rate of $\geq 80 \%$ defined as high adherence, and a rate of $<80 \%$ defined as low adherence, as reported previously. ${ }^{2,3}$ The average adherence rate, numbers of patients with high and low adherence, and adherence rate in each patient were compared between the 2 formulations. Safety was evaluated by assessing adverse events during the study period. The information on the enrolled patients was obtained from medical records.

\section{Questionnaires}

The English versions of the questionnaires are shown in Fig. 2 (the original was written in Japanese).

\section{Statistical Analysis}

All numerical values are shown as the median and range, or average \pm SD. Continuous variables were compared by $t$-tests, and proportions of categorical variables were compared by chi-square and Fisher exact tests. A $P$-value $\leq 0.05$ was considered statistically significant. Statistical analyses were performed using EZR version 1.33 (Saitama Medical Center, Jichi Medical University, Saitama, Japan).

\section{Ethical Considerations}

This study was conducted in accordance with the Declaration of Helsinki and with good clinical practice. The study protocol was approved by the Institutional Review Boards of Kitasato 
University Kitasato Institute Hospital and Kyorin University School of Medicine. Written informed consent was obtained from all participating patients.

\section{RESULTS}

\section{Patients}

A total of 49 patients were prospectively enrolled. Twelve patients were excluded, including 11 who did not attend within the scheduled period and 1 who chose to withdraw from the study. Thirty-seven patients therefore completed the study. To ensure an accurate comparison of the acceptabilities and efficacies of the 2 formulations, 4 patients were excluded from the final analysis (33 patients) because they did not receive doses of $4 \mathrm{~g} /$ day, almost all patients were in remission (partial Mayo score, $1.0 \pm 1.4$ ) (Table 1). There was no significant difference in any patient characteristics or questionnaire responses between the 2 groups, and we therefore assessed the acceptabilities of the 2 formulations without distinguishing between the groups.

\section{Formulation Acceptabilities}

The results of questionnaires 1 and 2 (Fig. 2) regarding the difficulties in taking each formulation are shown in Fig. 3. Significantly more patients considered the tablets difficult to take compared with the granules $(P=0.0005)$. When the 2 formulations were compared directly (questionnaire 3) (Fig. 2C), patients considered the granules to be significantly more acceptable than the tablets $(P=0.004)$ (Fig. 4A), largely due to the reduced volume of medication in the granule formulation (Fig. 4B).

\section{Medication Adherence}

The adherence rate in patients taking the tablets was $91 \% \pm 11 \%$ compared with $94 \% \pm 8 \%$ in patients taking the granules (Fig. 5 ). There was no significant difference between the adherence rates for the 2 formulations $(P=0.139)$, although adherence to the granules tended to be higher. Thirty percent of patients showed better adherence to the granules compared with the tablets, while $12 \%$ showed better adherence to the tablets $(P=0.180)$. Eighteen percent of patients missed taking tablets because of their acceptability, compared with only $3 \%$ who

\section{(A) Questionnaire 1 (about usability of the tablet) \\ Q I. Did you feel the tablets were hard to take? \\ Q II. In case you answered "yes" in Q I, why? (multiple answers are allowed) \\ $\begin{array}{lll}\text { 1. Size of the tablet } & \text { 2. Pill number } \quad 3 \text {. Frequency }\end{array}$ \\ 4. I do not like tablets not limited to the CR mesalazine 5. Tastes \\ 6. Portability 7. The other reason \\ Q III. How much percentage of dose did you take correctly?

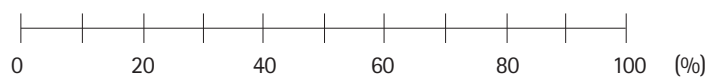 \\ Q IV. Have you missed the doses once or more often because of the usability of the tablets?}

B Questionnaire 2 (on the usability of the granules)
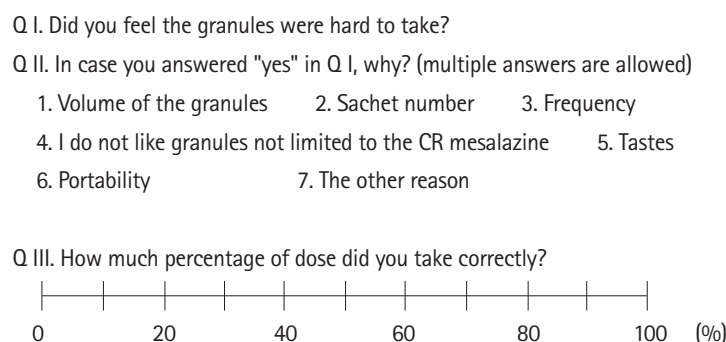

Q IV. Have you missed the doses once or more often because of the usability of the granules?
C Questionnaire 3 (on comparison of the tablets and the granules)

Q I. Which formulation did you feel easier to take?

Q II. In case you answered "tablet" in Q I. why? (multiple answers are allowed)

1. The volume of the granules was large

2. The sachet number of the granules was large

3. Taking granules is harder than tablets regardless of products

4. The tablets taste or smell better than the granules.

5. The tablets are easier to carry than the granules.

6. The other reason

Q III. In case you answered "granules" in Q I. why?

1. The size of the tablet is big

2. The tablet number is large

3. Taking granules is harder than tablets regardless of products

4. The granules taste or smell better than the tablets

5. The granules are easier to carry than the tablets

6. The other reason

Q V. Which formulation did you feel more effective?

Fig. 2. (A) Questionnaire 1: acceptability of tablets. (B) Questionnaire 2: acceptability of granules. (C) Questionnaire 3: comparison of tablets and granules. All the original questionnaires were written in Japanese. CR, controlled-release. 
Table 1. Patient Characteristics

\begin{tabular}{lcccc}
\hline Characteristics & All $(\mathrm{n}=\mathbf{3 3})$ & Group 1 $(\mathrm{n}=\mathbf{1 8})$ & Group 2 $(\mathrm{n}=\mathbf{1 5})$ & $P_{\text {-value }}$ a $^{\mathrm{a}}$ \\
\hline Age (yr) & $41 \pm 13$ & $40 \pm 13$ & $43 \pm 13$ & 0.675 \\
Sex (male/female) & $15 / 18$ & $10 / 8$ & $5 / 10$ & 0.296 \\
Disease duration (yr) & $9 \pm 9$ & $7 \pm 6$ & $12 \pm 11$ & 0.174 \\
Period of tablet (day) & $53 \pm 10$ & $55 \pm 6$ & $52 \pm 13$ & 0.342 \\
Period of granules (day) & $52 \pm 10$ & $52 \pm 10$ & $52 \pm 9$ & 0.801 \\
Concomitant medications & & & & - \\
$\quad$ Steroids & 0 & 0 & 0 & 0.458 \\
Thiopurines & 11 & 7 & 4 & 0.804 \\
Anti-TNF- $\alpha$ antibodies & 6 & 3 & 3 & 0.790 \\
$\quad$ Topical medications & 5 & 3 & 2 & 0.804 \\
Steroid-dependent & 6 & 3 & 3 & 0.698 \\
Steroid-refractory & 3 & 2 & 1 & 0.405 \\
Partial Mayo score at enrollment & $1.0 \pm 1.4$ & $1.2 \pm 1.6$ & $0.8 \pm 1.1$ & \\
\hline
\end{tabular}

Values are presented as mean \pm SD or number.

aroup 1 vs. group 2.

A

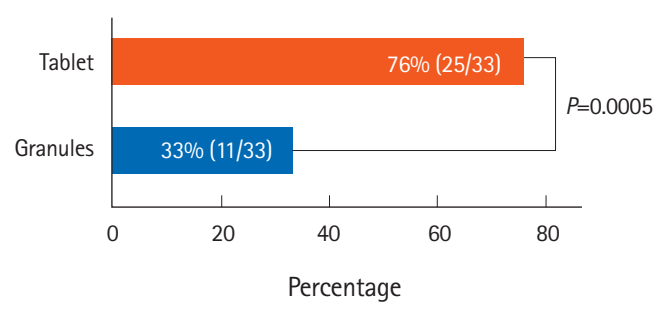

B

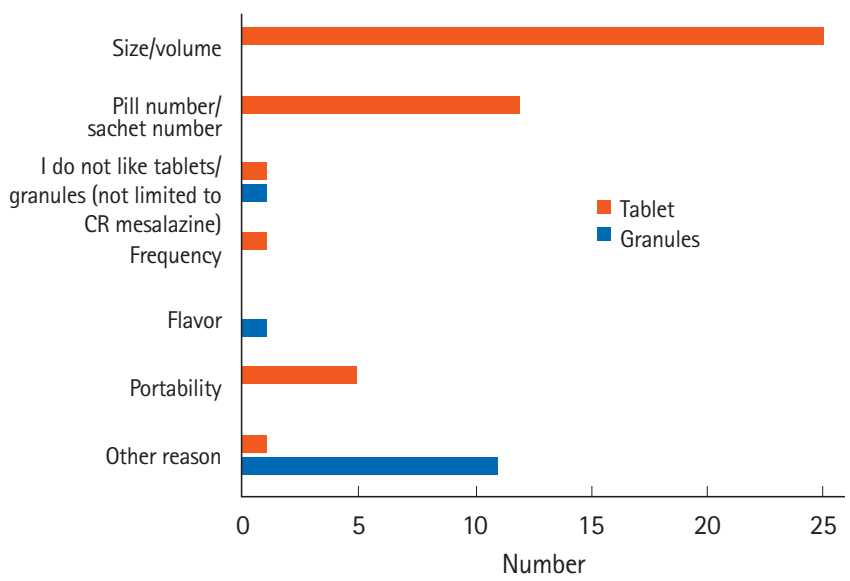

Fig. 3. Answers to the questions: (A) "Did you find the tablets/ granules difficult to take?" (questionnaires 1 and $2,0.1 ; n=33$, chi-square test, $P=0.0005)$, and (B) the reasons for the answers (multiple answers allowed). CR, controlled-release.

missed taking the granules $(P=0.0456)$. Eighteen percent also felt that the tablets were more likely to be missed than the gran-
A
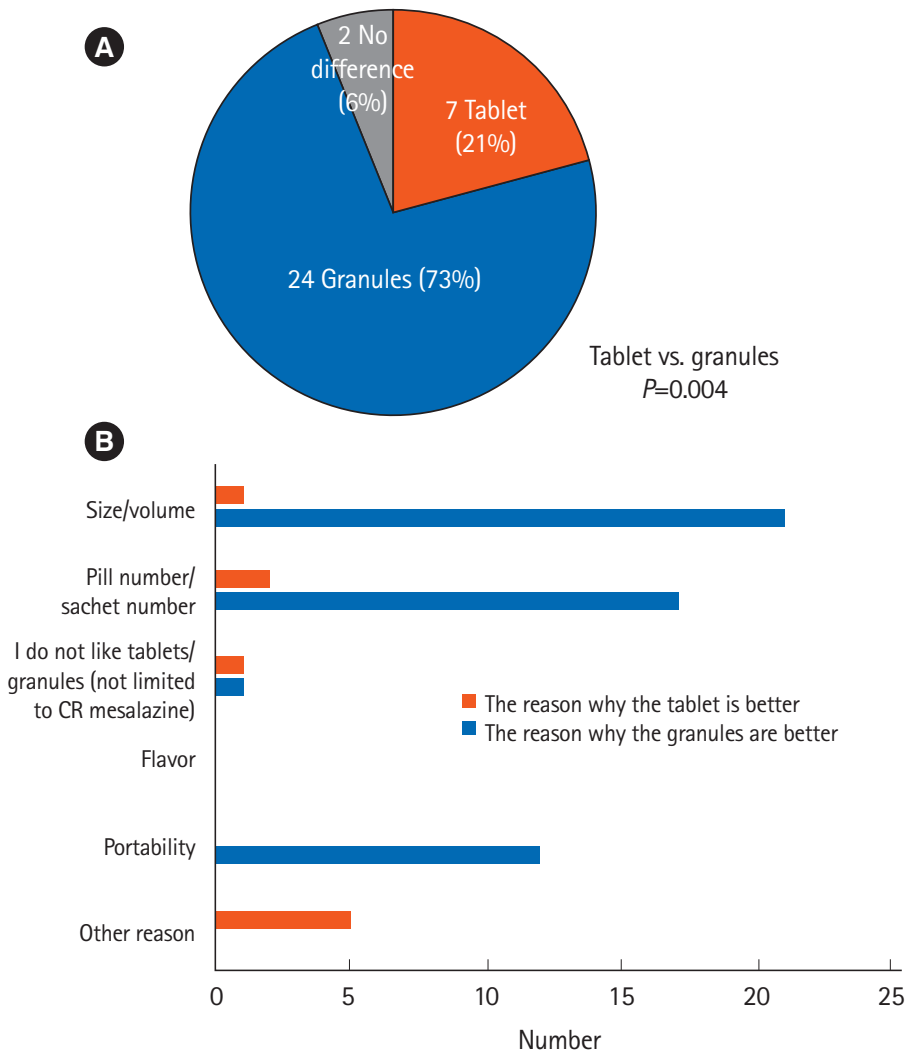

Fig. 4. Answers to the question: (A) "Which formulation did you find easier to take?" (questionnaire $3,0.1 ; n=33$, chi-square test, $P=0.004$ ), and ( $B$ ) the reasons for the answers (multiple answers allowed). CR, controlled-release.

ules, whereas no patient felt that the granules were more likely to be missed. Six percent of patients showed high adherence 


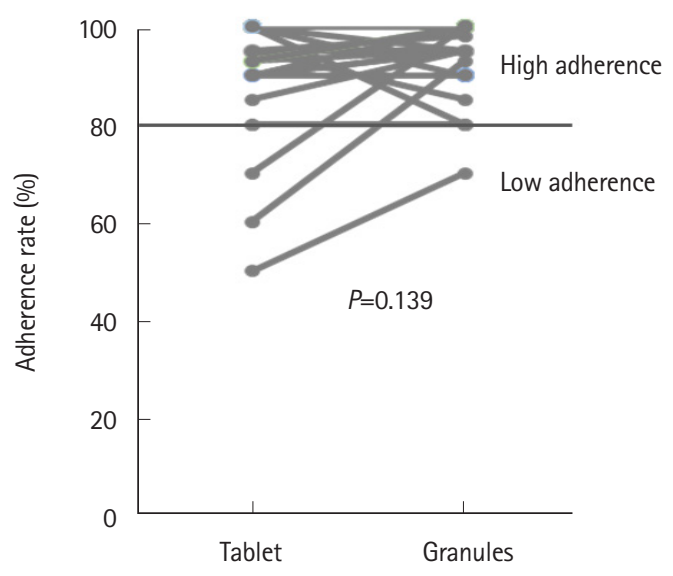

Fig. 5. Patient adherence rates to tablets and granules $(n=33$, paired $t$-test, $P=0.139$ ). High adherence, $\geq 80 \%$; low adherence, $<80 \%$.
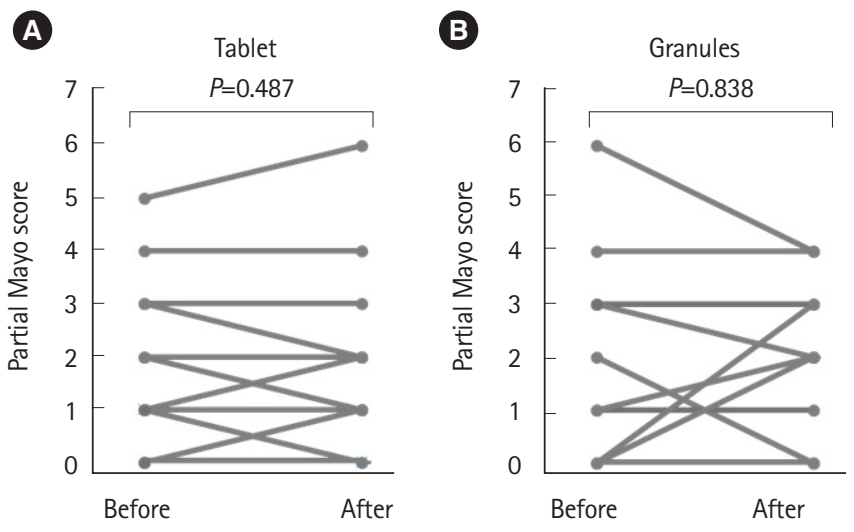

Fig. 6. Change of partial Mayo score before and after taking each formulation (no significant change was observed through this study. (A) Tablet ( $n=33, P=0.478)$ and (B) granules ( $n=33, P=0.838$ ).

to the granules but low adherence to the tablets.

\section{Efficacy}

Before and after taking each formulation, no significant change of partial Mayo score was observed in both the 2 formulations (Fig. 6). The responses regarding the patients' perceptions of formulation efficacy are shown in Fig. 7. A total of $70 \%$ of patients noticed no difference between the 2 formulations, while $18 \%$ and $12 \%$ considered the granules and tablets to be more effective, respectively. There was no significant difference between the 2 formulations in terms of efficacy.

\section{Adverse Events}

Most patients experienced no adverse events related to CR mesalazine during the study period. One patient who had taken CR mesalazine tablets before enrollment experienced de-

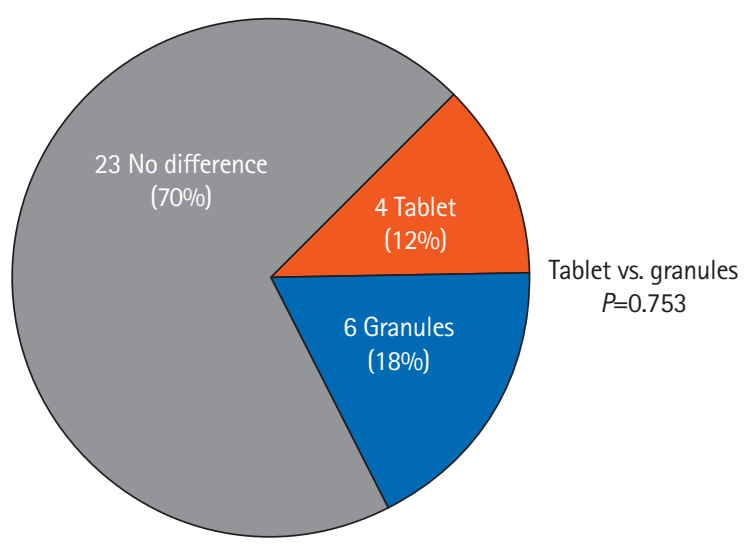

Fig. 7. Answers to the question "Which formulation did you feel was more effective?" (questionnaire $3,0 . V ; n=33$, chi-square test, $P=0.753$ ).

terioration of abdominal symptoms during the granule period and improved after switching to the tablets; the partial Mayo score of the patient was 0 at enrollment, increased to 3 after taking the granules and decreased to 2 after taking the tablets.

\section{DISCUSSION}

To the best of our knowledge, this was the first study to compare the acceptability, adherence, and efficacy of 2 different formulations of CR mesalazine directly, using a crossover design to avoid potential bias. We demonstrated that CR mesalazine granules were more acceptable than tablets, and patient adherence tended to be slightly better for granules than for tablets, although there was no statistically significant difference.

The questionnaire responses revealed that many patients found the size and number of the tablets unpleasant. It is necessary to take 8 or 16 (500 or $250 \mathrm{mg}$, respectively) CR mesalazine tablets to take in $4 \mathrm{~g}$ of mesalazine. The situation is similar for other mesalazine formulations, such as $\mathrm{pH}$-dependent mesalazine $\left(\right.$ Asacol $^{\circledR}$ ) (e.g., 10 tablets of $400 \mathrm{mg}$ to administer $4 \mathrm{~g}$ mesalazine). Furthermore, the relatively large volume of CR mesalazine tablets, due to the large additive content, was also a major reason for the patient dissatisfaction. CR mesalazine granules were therefore preferable because of both their formulation and the reduced volume required.

The acceptability of the granules was significantly superior to that of the tablets, with approximately three-quarters of patients considering the granules to be preferable to the tablets by the end of this crossover study. Interestingly, the granule 
formulation was preferred not only because of the reduced pill burden in terms of volume and number, but also because of its portability. Medication portability may be an important factor for patients who need to take their medications outside their homes during their daily life, and patients may hesitate or forget to carry medications with them because of poor portability. Granules might thus be a good option for patients who take their medications outside their home.

Although the acceptabilities of the 2 formulations differed, their average adherence rates were not significantly different. The frequency of taking medications has been identified as one of the most important factors affecting adherence in patients with various diseases, including IBD. ${ }^{10-12}$ In this study, the frequency of intake was the same for both formulations, which may help to explain why the average adherence rates were similar. However, the study design may have led to differences in adherence between the formulations being underestimated. It is difficult to assess real-world adherence in prospective studies, because patients may pay more attention to the protocol and/or overestimate their adherence under trial settings. In fact, the adherence rates in this study were very high (granules $95 \%$, tablet $91 \%$ ) compared with the previous reports. ${ }^{2,3}$ In addition, we did not assess long-term adherence in this shortterm study, and it is possible that long-term adherence to the granules might be superior to that of the tablets in a real-world situation, because of significantly better acceptance.

The short-term nature of this study also limited the efficacy evaluation, and a longer observation period may be needed to assess the difference in efficacy in terms of maintaining remission. Adherence guidelines produced by the National Collaborating Centre for Primary Care suggest that although there is no convincing evidence that changes in drug formulation improve adherence, the number, taste, smell, size, and shape of the pills might nonetheless affect medication adherence. ${ }^{13}$ Kane et al. ${ }^{2}$ also reported that $30 \%$ of UC patients did not take their medication because of the large number of pills, and a lower adherence rate was associated with a higher risk of future clinical relapse. These findings suggest that adherence declines with lower acceptability of the medication in some patients, possibly leading to a flare-up. Indeed, $6 \%$ of patients in the current study showed high adherence to the granules but low adherence to the tablets, and all said that they had missed a dose at least once because of the poor acceptability of the tablets. Interestingly, a lower pill burden was also reported to be associated with better adherence and virological suppression in patients with human immunodeficiency virus in- fection, which also requires good adherence to the daily medication, and may be associated with treatment fatigue after longterm treatment. ${ }^{14} \mathrm{UC}$ treatment has similar characteristics from the aspect of long-term maintenance, and the obvious difference in acceptability in the present study may thus have an important impact on the long-term treatment outcomes in patients with UC.

In conclusion, CR mesalazine granules are a highly acceptable formulation of 5-ASA, and may be associated with better long-term outcomes than tablets as a result of improved patient adherence to the medication.

\section{FINANCIAL SUPPORT}

The authors received no financial support for the research, authorship, and/or publication of this article.

\section{CONFLICT OF INTEREST}

TK received lecture fees from Mitsubishi-Tanabe Pharma, Eisai, Kyorin Pharmaceutical, Abbvie, Janssen, JIMRO, Ajinomoto Pharma, EA Pharma, Astellas, Mochida Pharmaceutical, Asahi Kasei Medical, Takeda Pharmaceutical, Gilead Sciences, Celltrion, Nippon Kayaku, and Alfresa Pharma, advisory/consultancy fees from Janssen, Pfizer, Kyorin Pharmaceutical, Mochida Pharmaceutical, Takeda Pharmaceutical, Eli Lilly, Ferring Pharmaceuticals, Nippon Kayaku, Thermo Scientific, and Covidien, and research grants from EA Pharma, Thermo Scientific, and Alfresa Pharma. KY received lecture fees from Eisai, Mitsubishi-Tanabe Pharma, EA Pharma, Abbvie, and Pfizer and advisory fees from Janssen, EA Pharma, and Mochida Pharmaceutical. RO received lecture fees from ZERIA and Pfizer Pharmaceutical Co., Ltd. MN received consulting fees from Medtronic Co., Ltd., Takeda Pharmaceutical, Mochida Pharmaceutical, and ZERIA Pharmaceutical. ES received lecture fees from Abbvie, Ajinomoto Pharma, and EA Pharma, chairmanship etc. fees from Abbvie, EA Pharma, and Mitsubishi-Tanabe Pharma, and research grants from EA Pharma, Abbvie, JIMRO, and ZERIA Pharma. TaH received lecture fees from Mitsubishi-Tanabe Pharma, EA Pharma, Kyorin Pharmaceutical, Abbvie, Janssen, JIMRO, Mochida Pharmaceutical, Takeda Pharmaceutical, Gilead Sciences, and Nippon Kayaku, advisory/consultancy fees from Janssen, Pfizer, Kyorin Pharmaceutical, Mochida Pharmaceutical, Takeda Pharmaceutical, Eli Lilly, Nichi-Iko Pharmaceutical Co., Ltd., and Celgene, and research grants from Mitsubishi-Tanabe Pharma, EA Phar- 
ma, Kyorin Pharmaceutical, Abbvie, JIMRO, Mochida Pharmaceutical, Takeda Pharmaceutical, Nippon Kayaku, Daiichi Sankyo Co. Ltd., Astellas Pharma Inc., Mylan EPD, and Boston Scientific. ToH received lecture fees from Abbvie Inc., Kyorin Pharmaceutical Co., Ltd., Eisai Co., Ltd., Mitsubishi Tanabe Pharma Co., Ltd., EA Pharma Co., Ltd., JIMRO Co., Ltd., and ZERIA Pharmaceutical Co., Ltd. No funding was received for this work from any of the organizations.

\section{AUTHOR CONTRIBUTION}

Conceptualization: Yagisawa K, Kobayashi T. Methodology: Yagisawa K, Kobayashi T. Formal analysis: Yagisawa K, Kobayashi T. Project administration: Kobayashi T. Visualization: Yagisawa K. Writing-original draft: Yagisawa K, Kobayashi T. Writing-review and editing: Ozaki R, Hisamatsu T, and Hibi T. Approval of final manuscript: all authors.

\section{ACKNOWLEDGEMENTS}

We are grateful to Tadae Mori, Toyomi Ishibashi, and Yuki Watanabe for helping us to accomplish this study. We also thank Susan Furness, PhD, from Edanz Group (www.edanzediting. $\mathrm{com} / \mathrm{ac}$ ) for editing a draft of this manuscript.

\section{REFERENCES}

1. Magro F, Gionchetti P, Eliakim R, et al. Third European evidence-based consensus on diagnosis and management of ulcerative colitis. Part 1: definitions, diagnosis, extra-intestinal manifestations, pregnancy, cancer surveillance, surgery, and ileo-anal pouch disorders. J Crohns Colitis 2017;11:649-670.

2. Kane S, Huo D, Aikens J, Hanauer S. Medication nonadherence and the outcomes of patients with quiescent ulcerative colitis. Am J Med 2003;114:39-43.

3. Kawakami A, Tanaka M, Nishigaki M, et al. Relationship between non-adherence to aminosalicylate medication and the risk of clinical relapse among Japanese patients with ulcerative colitis in clinical remission: a prospective cohort study. J Gastroenterol 2013;48:1006-1015.
4. Hanauer S, Schwartz J, Robinson M, et al. Mesalamine capsules for treatment of active ulcerative colitis: results of a controlled trial. Pentasa Study Group. Am J Gastroenterol 1993; 88:1188-1197.

5. Flourié B, Hagège H, Tucat G, et al. Randomised clinical trial: once- vs. twice-daily prolonged-release mesalazine for active ulcerative colitis. Aliment Pharmacol Ther 2013;37:767-775.

6. Wilding IR, Kenyon CJ, Hooper G. Gastrointestinal spread of oral prolonged-release mesalazine microgranules (Pentasa) dosed as either tablets or sachet. Aliment Pharmacol Ther 2000;14:163-169.

7. Devlen J, Beusterien K, Yen L, Ahmed A, Cheifetz AS, Moss AC. Barriers to mesalamine adherence in patients with inflammatory bowel disease: a qualitative analysis. J Manag Care Spec Pharm 2014;20:309-314.

8. Kawakami A, Tanaka M, Ochiai R, et al. Difficulties in taking aminosalicylates for patients with ulcerative colitis. Gastroenterol Nurs 2012;35:24-31.

9. Severs M, Zuithoff PN, Mangen MJ, et al. Assessing self-reported medication adherence in inflammatory bowel disease: a comparison of tools. Inflamm Bowel Dis 2016;22:2158-2164.

10. Ford AC, Khan KJ, Sandborn WJ, Kane SV, Moayyedi P. Oncedaily dosing vs. conventional dosing schedule of mesalamine and relapse of quiescent ulcerative colitis: systematic review and meta-analysis. Am J Gastroenterol 201 1;106:2070-2077.

11. McLaughlin T, Hogue SL, Stang PE. Once-daily bupropion associated with improved patient adherence compared with twice-daily bupropion in treatment of depression. Am J Ther 2007;14:221-225.

12. Amara W, Antoniou S. Benefits of once-daily dosing with nonvitamin $\mathrm{K}$ antagonist oral anticoagulants in patients with atrial fibrillation. Eur Heart J Suppl 2016;18(Suppl D):D1-D6.

13. National Collaborating Centre for Primary Care (UK). Medicines adherence: involving patients in decisions about prescribed medicines and supporting adherence. London: Royal College of General Practitioners (UK); 2009.

14. Nachega JB, Parienti JJ, Uthman OA, et al. Lower pill burden and once-daily antiretroviral treatment regimens for HIV infection: a meta-analysis of randomized controlled trials. Clin Infect Dis 2014;58:1297-1307. 